\title{
RHINOLOGY
}

\section{Effects of biological therapies on chronic rhinosinusitis in severe asthmatic patients}

\author{
L'efficacia delle terapie biologiche sulla rinosinusite cronica nel paziente asmatico severo
}

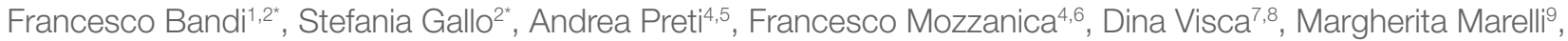
Enrico Maddalone ${ }^{2}$, Cinzia Gambarini ${ }^{9}$, Adriano Vaghi ${ }^{10}$, Antonio Spanevello ${ }^{7,8}$, Paolo Castelnuovo ${ }^{2,3}$

${ }^{1}$ Department of Otolaryngology-Head and Neck Surgery, European Institute of Oncology, Milan, Italy; ${ }^{2}$ Department of Otorhinolaryngology, University of Insubria and ASST Sette Laghi, Varese, Italy; ${ }^{3}$ Department of Biotechnology and Life Sciences, University of Insubria, Varese, Italy; ${ }^{4}$ Department of Otorhinolaryngology, IRCCS Multimedica, Milano, Italy; ${ }^{5}$ Department of Medicine and Surgery, University of Insubria, Varese, Italy; ${ }^{6}$ Department of Biomedical and Clinical Sciences, University of Milan, Italy; ${ }^{7}$ Istituti Clinici Scientifici Maugeri IRCCS, Respiratory Rehabilitation of the Institute of Tradate, Italy; ${ }^{8}$ Department of Medicine and Surgery, Respiratory Diseases, University of Insubria, Varese-Como, Italy; ${ }^{9}$ Department of Pulmonology, ASST Sette Laghi, Varese, Italy; ${ }^{10}$ ASST-Rhodense, UOC Pneumologia, Garbagnate Milanese, Milan, Italy

F. BandiandS. Gallo contributed equally to the work.
\end{abstract}

\section{SUMMARY}

Objective. The introduction of monoclonal antibody $(\mathrm{mAb})$ therapies represents a promising treatment for refractory chronic rhinosinusitis (CRS). We assessed the effects of selected mAbs (omalizumab, mepolizumab, benralizumab) on CRS in severe asthmatic patients in a real-life setting. Methods. A prospective observational study on severe asthmatic patients, treated with 3 different mAb (omalizumab, mepolizumab, benralizumab), and comorbid CRS was conducted. All patients were followed for 52 weeks. The degree of nasal control, SinoNasal Outcome Test (SNOT) 22, Nasal Polyp Score (NPS), Lund Kennedy Score (LKS) were collected at baseline and at 52-week.

Results. 40 patients (33 with nasal polyps) were studied. 33 patients $(82.5 \%$ ) had uncontrolled nasal disease at baseline, and $15(37.5 \%)$ were uncontrolled after 52 weeks. Significant improvement was observed for SNOT 22 ( P < 0.001), SNOT 1-12 $(\mathrm{P}<0.001)$ and degree of nasal control $(\mathrm{P}<0.001)$. Differences in NPS $(\mathrm{P}=0.130)$ and LKS $(\mathrm{P}=0.124)$ were not significant. Net change in the above-mentioned parameters among the three treatment groups was not significantly different.

Conclusions. The study shows an improvement of nasal symptoms after 52 weeks of mAb treatment, which was not associated with significant improvement of endoscopic findings. Larger studies are needed to assess the real-life efficacy of mAbs in CRS.

KEY WORDS: chronic rhinosinusitis, nasal polyps, asthma, monoclonal antibody, biological therapies

\section{RIASSUNTO}

Obiettivi. L'introduzione degli anticorpi monoclonali $(m A b)$ rappresenta una promettente risorsa per il trattamento delle rinosinusiti croniche (RSC) refrattarie. Obiettivo dello studio è valutare l'efficacia del trattamento biologico sulla RSC nel paziente asmatico severo in un contesto real-life.

Metodi. È stato condotto uno studio osservazionale di 52 settimane su pazienti asmatici severi, trattati con 3 diversi mAb (omalizumab, mepolizumab, benralizumab), e con RSC come comorbidità. Sono stati raccolti raccolti dati riguardanti il grado di controllo nasale, il SinoNasal Outcome Test (SNOT) 22, il Nasal Polyp Score (NPS), il Lund Kennedy Score (LKS) al baseline e a 52 settimane.

Risultati. Sono stati analizzati 40 pazienti (33 con polipi nasali). 33 pazienti ( $82.5 \%$ ) presentavano una RSC non controllata al baseline, 15 (37.5\%) soffrivano ancora di RSC non controllata dopo 52 settimane. Un miglioramento significativo è descritto per lo SNOT 22 $(P<0.001)$, lo SNOT 1-12 $(P<0.001)$ e il grado di controllo nasale $(P<0.001)$; differenze nel NPS $(P=0.130)$ e LKS $(P=0.124)$ sono risultate non significative. Il cambiamento netto dei sopracitati parametri tra i tre gruppi di trattamento non era significativamente differente.
Received: February 26, 2020

Accepted: September 6, 2020

Correspondence

Bandi Francesco

Department of Otolaryngology-Head and Neck Surgery, European Institute of Oncology (IEO) via Ripamonti 435, 20141 Milano

Tel. +3902 57489490. Fax +390294379216

E-mail: bandi.francesco@gmail.com

Funding

None.

Conflict of interest

The Authors declare no conflict of interest.

How to cite this article: Bandi F, Gallo S, Preti A, et al. Effects of biological therapies on chronic rhinosinusitis in severe asthmatic patients. Acta Otorhinolaryngol Ital 2020;40:435-443. https:// doi.org/10.14639/0392-100X-N0716

() Società Italiana di Otorinolaringoiatria e Chirurgia Cervico-Facciale

\section{(c) (1) $(9)$}

This is an open access article distributed in accordance with the CC-BY-NC-ND (Creative Commons Attribution-NonCommercial-NoDerivatives 4.0 International) license. The article can be used by giving appropriate credit and mentioning the license, but only for non-commercial purposes and only in the original version. For further information: https:// creativecommons.org/licenses/by-nc-nd/4.0/deed.en 
Conclusioni. Lo studio evidenzia a 52 settimane di trattamento con mAb un miglioramento dei sintomi, non associato a cambiamenti significativi dei riscontri endoscopici. Sono necessari studi più ampi per determinare l'efficacia nella real-life dei mAb nella RSC.

PAROLE CHIAVE: rinosinusite cronica, polipi nasali, asma, anticorpi monoclonali, terapie biologiche

\section{Introduction}

Chronic rhinosinusitis (CRS) is a heterogeneous inflammatory disease with an as-yet-undefined aetiology. A complex combination of altered immunity, genetics and environmental factors (microbiome, allergy, etc.) seems to play a cooperative role in disease initiation and progression ${ }^{1}$. Going beyond its rooted phenotypes (with nasal polyps, CRSwNP; without nasal polyps, CRSsNP), CRS is nowadays considered an umbrella term for different clinical entities mirroring diverse biomolecular inflammatory processes, the endotypes ${ }^{2}$. Extensive knowledge on CRS pathophysiological mechanisms would be entirely speculative, except endotypes become a potential therapeutic target.

Since CRS is closely connected to asthma by the "unified airways" theory ${ }^{3}$, it is no surprise that not only pathogenesis, but also therapeutic principles may be similar in both sections of the airways. Thus, biological therapies currently approved for severe asthma may represent a treatment option for refractory CRS cases, who can count only on courses of oral corticosteroids (OCS), antibiotics or repeated sinus surgeries, with failures ranging from 20 to $40 \%$ according to different series ${ }^{4,5}$.

The efficacy of biological agents in the treatment of asthma is widely reported and documented in literature ${ }^{6}$; meanwhile several research studies are documenting encouraging results for CRS, as well ${ }^{7,8}$. In Italy, 3 monoclonal antibodies $(\mathrm{mAb})$ are approved for the treatment of severe asthma in clinical practice: omalizumab (anti-IgE), mepolizumab (anti-IL5) and benralizumab (anti-IL5R). Phase III trials are currently evaluating the efficacy of the same mAbs in patients affected by CRSwNP. Therefore, to date, in Italy, the indication to start a biological therapy falls in the purview of pneumological practice and dependent on the coexistence of severe asthma.

While several randomised clinical trials (RCT) have highlighted the efficacy of mAbs in CRSwNP ${ }^{9-12}$, the current literature still lacks observational studies evaluating the response to biological treatments in real-life.

Therefore, while waiting to actively take part, as rhinologists, in treatment indications and selection of biological therapies, we report our preliminary real-life data regarding the clinical effects of selected mAbs (omalizumab, mepolizumab and benralizumab) on CRS in adult patients treated for severe asthma. The primary endpoint was to portray the main clinical CRS-related characteristics of a cohort of patients with severe asthma treated with biologicals and with CRS as a comorbidity. Secondary endpoints included evaluation of endoscopic sinonasal changes, variations in patient-reported outcomes and in the degree of nasal disease control over 52 weeks of treatment.

\section{Materials and methods}

\section{Study population}

An observational study was conducted at the ENT Department of a single tertiary care centre enrolling subjects affected by severe uncontrolled asthma, eligible for $\mathrm{mAb}$ therapy, and with CRS as a comorbidity between July 2017 and May 2019. The study was concluded in June 2020.

Included patients were over 18 years old, with a negative clinical history for genetic syndromes, congenital or acquired immunodeficiency, autoimmune disorders, malignancy or history of head and neck cancer, and drug abuse. Patients were addressed by pulmonologists to specific biological agents (anti-IgE, omalizumab; anti-IL5, mepolizumab; anti-IL5R, benralizumab) to treat severe asthma according to GINA guidelines ${ }^{13-16}$ within 1 month from our baseline evaluation and were monitored with regular clinical and endoscopic assessments every 3 months. For analytical reasons and in order to align data, the 52-week visit after first mAb dosing was set as a follow-up cut-point.

Rhinologists involved in the study did not intervene in treatment selection which depended exclusively on features of asthma. We point out that all patients presented a type 2 inflammation profile of the airways, defined by blood eosinophil count $\geq 250$ cells $/ \mathrm{mm}^{3}$ and/or peripheral total $\operatorname{IgE}$ $\geq 100 \mathrm{kU} / \mathrm{L}$ at the time of enrollment ${ }^{17}$.

The study was conducted in compliance with the Helsinki Declaration and with policies approved by the Insubria Board of Ethics. Informed consent was obtained from all participants included in the study.

\section{Study methods}

CRS was confirmed and classified as controlled, partly controlled or uncontrolled according to EPOS guidelines ${ }^{17,18}$.

Data concerning demographic features, CRS and asthma onset, smoking habits, non-steroidal anti-inflammatory drug (NSAID) intolerance, blood eosinophil count, peripheral IgE count, nasal therapies, need for OCS for airways exacerbations, previous nasal surgeries, adverse events (AEs) were collected in an electronic database ${ }^{19}$. 
Sensitization for common inhalants was evaluated on the basis of recent pre-existing skin or blood tests as defined by the Global Atlas Test Panel for Europe ${ }^{20}$.

Symptoms were collected through the Italian version of the SinoNasal Outcome Test 22 (SNOT 22) questionnaire ${ }^{21}$ and analysed both as total (SNOT 22) and partial (SNOT 1-12) scores, and as individual symptoms ${ }^{22,23}$.

Endoscopic findings of the entire cohort were scaled according to the Lund-Kennedy Score (LKS) ${ }^{24}$ and the size of nasal polyps in the CRSwNP subgroup was calculated through the Nasal Polyp Score (NPS) ${ }^{25}$.

Patients were further sorted into three different subgroups depending on the type of mAb therapy (anti-IgE, anti-IL5 and anti-IL5R groups) and reassessed by means of the above-mentioned outcome measures.

\section{Statistical analysis}

The Kolmogorov-Smirnov test was used to check the normality of the distribution. Since this test demonstrated that the distribution was not normal, non-parametric tests were used. In particular, the median and interquartile ranges were calculated for all variables. The Mann-Whitney test and Fisher test were used to compare the distribution of continuous and non-continuous variables between the pre and post-treatment periods and between the three treatment groups. For all statistical comparisons an $\alpha=0.05$ was used.

\section{Results}

A cohort of 40 severe asthmatic patients was examined. 33 patients $(82.5 \%)$ were affected by CRSwNP, and 7 patients $(17.5 \%)$ were affected by CRSsNP. Demographic data are reported in Table I.

Eighteen patients $(45 \%)$ were in continuative or intermittent OCS treatment for control of airway exacerbations; 30 patients $(75 \%)$ had a history of endoscopic sinus surgery and the most frequent procedure was full-house endoscopic sinus surgery with removal of middle turbinate (ESS) (13 patients, $43.3 \%$ ). 20 subjects (66.7\%) underwent more than 1 endoscopic procedure, with a mean of 2.9 surgeries per patient. All operated patients performed a sinus CT scan before the last surgery and the mean Lund-Mackay score was $16.5 \pm 4.0$ (range 6-22). The majority of operated patients (24 patients, $80 \%$ ) underwent the last surgery before the introduction of the mAb; in 6 subjects (20\%) surgery was performed during $\mathrm{mAb}$ treatment. Further details on surgical treatments are specified in Table II.

Mean baseline LKS was $5.4 \pm 2.7$ (median 5, IQR 4-7) and mean 52-week LKS was $4.8 \pm 3.1$ (median 4, IQR 3-6.2). In the CRSwNP subgroup, mean baseline NPS was
Table I. Demographic data of the cohort $(n=40)$.

\begin{tabular}{lc} 
Variable & Value \\
Age (years) & $54.4($ range $31-73)$ \\
Sex, M:F & $12(30 \%): 28(70 \%)$ \\
Smoke & \\
Active & $2 / 40(5 \%)$ \\
Ex-smoker & $12 / 40(30 \%)$ \\
Non smoker & $26 / 40(65 \%)$ \\
Inhalant sensitisation & $29 / 40(72.5 \%)$ \\
Seasonal & $3 / 29(10.3 \%)$ \\
Perennial & $8 / 29(27.6 \%)$ \\
Both & $18 / 29(62.1 \%)$ \\
NSAID intolerance & $11 / 40(27.5 \%)$ \\
CRSwNP & $33 / 40(82.5 \%)$ \\
CRSsNP & $7 / 40(17.5 \%)$ \\
CRS onset & \\
Early (<40 y) & $20 / 40(50 \%)$ \\
Late ( $\geq 40$ y) & $20 / 40(50 \%)$ \\
Asthma onset & \\
Early (< 40 y) & $17 / 40(42.5 \%)$ \\
Late ( $\geq 40$ y) & $23 / 40(57.5 \%)$ \\
CRS vs asthma onset & \\
Concordant & $21 / 40(52.5 \%)$ \\
CRS followed by asthma & $4 / 40(10 \%)$ \\
Asthma followed by CRS & $15 / 40(37.5 \%)$ \\
\hline M: male; F: female; NSAID: non-steroidal anti-inflammatory drugs; CRSwNP: chronic \\
rhinosinusitis with nasal polyps; CRSsNP: chronic rhinosinusitis without nasal polyps; y: \\
years old; CRS: chronic rhinosinusitis & \\
&
\end{tabular}

Table II. Surgical history of the cohort.

\begin{tabular}{lc} 
Number of operated patients & $30 / 40(75 \%)$ \\
Type of surgery & \\
Polypectomy & $5 / 30(16.7 \%)$ \\
Anterior FESS & $2 / 30(6.7 \%)$ \\
FESS & $6 / 30(20 \%)$ \\
ESS & $13 / 30(43.3 \%)$ \\
ESS + Draf III frontal sinusotomy & $4 / 30(13.3 \%)$ \\
Mean number of surgeries for each patient & $2.9($ range $1-13)$ \\
Mean age at first surgery & $42.1($ range $18-59)$ \\
Last surgery performed before mAb & $24 / 30(80 \%)$ \\
Last surgery performed during mAb & $6 / 30(20 \%)$ \\
\hline
\end{tabular}

FESS: functional endoscopic sinus surgery; ESS: endoscopic sinus surgery; $m A b$ : monoclonal antibody

$2.6 \pm 1.9$ (median 2, IQR 1.2-4) and mean 52-week NPS was $2.0 \pm 2.1$ (median 2, IQR 0-2.7). Differences between scores at baseline and at 52-week were not significant at Mann-Whitney test with $\mathrm{P}=0.124$ for LKS and $\mathrm{P}=0.130$ for NPS.

The mean baseline SNOT 22 score was $56.4 \pm 27.3$ (range 8-104; median 57,5, IQR 35.7-80) and SNOT 1-12 was $33.1 \pm 14.5$ (range 4-59; median 35, IQR 24-45.2). The 
mean 52-week SNOT 22 score was $30.5 \pm 21.2$ (range 1-83; median 26.5, IQR 14-47) and SNOT 1-12 was $17.5 \pm 11.7$ (range 0-46; median 17.5, IQR 6.7-26). Differences between scores at baseline and at 52-week were found significant at Mann-Whitney test with $\mathrm{P}<0.001$ for SNOT 22 score and $\mathrm{P}<0.001$ for SNOT 1-12 score. Statistically significant differences were also found for individual sinonasal symptoms such as nasal blockage $(\mathrm{P}<0.001)$, rhinorrhoea $(\mathrm{P}<0.001)$, hyposmia $(\mathrm{P}=0.023)$, facial pain $(\mathrm{P}=0.027)$ and ear fullness $(\mathrm{P}=0.006)$.

Nasal disease at baseline was partly controlled in 7 patients $(17.5 \%)$ and uncontrolled in 33 patients $(82.5 \%)$. No patient was controlled at baseline. At 52-weeks, nasal disease was controlled in 6 patients (15\%), partly controlled in 19 $(47.5 \%)$ and uncontrolled in 15 (37.5\%). In detail, the nasal disease score remained unchanged in 20 patients $(50 \%)$, irrespective of baseline status (15 uncontrolled, 5 partly controlled), whereas 20 patients (50\%) clinically improved with 16 patients climbing one rank of the nasal disease scale and 4 patients climbing two ranks. Overall, a positive behavioural trend is evident $(\mathrm{P}<0.001)$.

No serious AEs were reported in our cohort. However, 25 patients $(62.5 \%)$ reported minor AEs. Complaints included nasopharyngitis $(17 / 40,42.5 \%)$, oropharyngeal pain (15/40, $37.5 \%)$, back pain $(11 / 40,27.5 \%)$, arthralgia $(10 / 40,25 \%)$, influenza $(8 / 40,20 \%)$ and pyrexia $(2 / 40,5 \%)$.

Subgroup analysis: anti-IgE, anti-IL5 and anti-IL5R groups Eleven patients $(27.5 \%)$ were addressed to omalizumab therapy, $20(50 \%)$ to mepolizumab and the remaining 9 $(22.5 \%)$ to benralizumab. Seven patients $(17.5 \%)$ had a history of a previous $\mathrm{mAb}$ treatment. In particular, 5 patients, first selected for omalizumab after an average of $26.4 \pm 15.6$ months were switched to mepolizumab due to poor response on the lower airways; for the same reason, 2 patients suspended mepolizumab after $5.0 \pm 1.4$ months and started benralizumab for unsuccessful control of asthma symptoms.

Demographics of treatment groups are shown in Table III. Differences concerning inhalants sensitisation $(P=0.196)$, NSAID intolerance $(\mathrm{P}=0.411)$, prevalence of nasal polyps $(\mathrm{P}=0.892)$, $\mathrm{CRS}$ onset $(\mathrm{P}=0.818)$, asthma onset $(P=0.625)$, endoscopic sinus surgeries $(P=0.431)$, chronic OCS treatment $(\mathrm{P}=0.638)$ and nasal steroid treatment $(\mathrm{P}=0.258)$ were not significantly different.

Differences concerning parameters as SNOT scores and individual symptoms, LKS, NPS and blood eosinophil count between baseline and at 52-week in each treatment group are shown in Table IV.

Briefly, improvements in SNOT 22 and SNOT 1-12 scores were evident in all three groups, with anti-IgE and antiIL5 groups reaching a significant difference between baseline and 52 weeks for both parameters. Moreover, anti-IL5 significantly reduced nasal blockage $(\mathrm{P}=0.001)$ and ear fullness $(\mathrm{P}=0.026)$ scores, whereas anti-IL5R significantly reduced the rhinorrhoea score $(\mathrm{P}=0.040)$.

A significant change was observed in nasal disease control at 52 weeks in all three treatment groups (Fig. 1).

Concerning endoscopic parameters, no significant variations were evident for LKS between baseline and 52 weeks in any treatment group. Only anti-IL5 significantly reduced NPS in the subgroup of patients with nasal polyps $(\mathrm{P}=0.029)$. However, once the 3 patients on treatment with mepolizumab and operated on during the 52-week observation period were excluded from the analysis, this significance was lost $(\mathrm{P}=0.096)$.

Blood eosinophil count was available at baseline for 37 patients and at 52 weeks for only 29 patients. No differ-

Table III. Characteristics and differences between treatment groups. P is calculated with a non-parametric Mann-Whitney test.

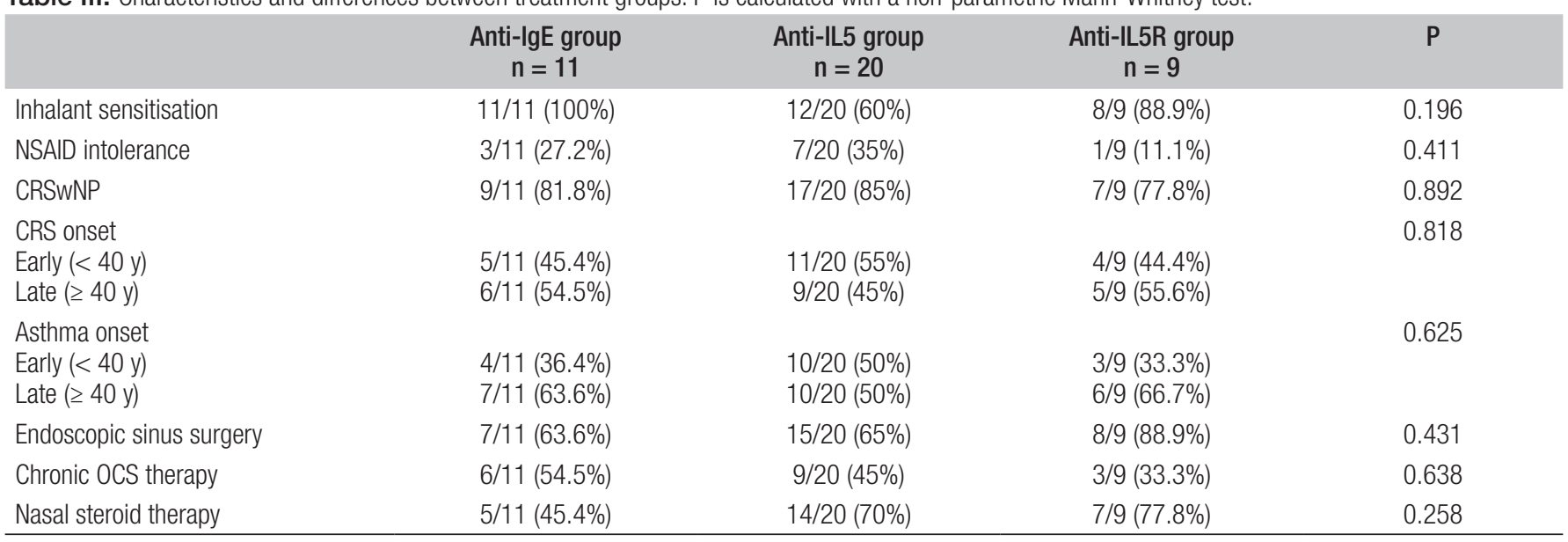

NSAID: non-steroidal anti-inflammatory drugs; CRSwNP: chronic rhinosinusitis with nasal polyps; CRS: chronic rhinosinusitis; y: years old; OCS: oral corticosteroids 
Table IV. Differences between baseline and 52-week measurements of selected variables in each treatment group. $P$ is calculated with a non-parametric Mann-Whitney test.

\begin{tabular}{|c|c|c|c|}
\hline \multirow[t]{3}{*}{ 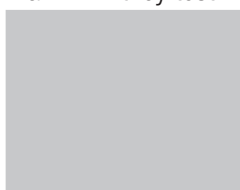 } & \multicolumn{3}{|c|}{$\begin{array}{l}\text { Anti-lgE group } \\
\quad \mathrm{n}=11\end{array}$} \\
\hline & Baseline & 52-week & $P$ \\
\hline & \multicolumn{2}{|c|}{ median (IQR $\left.25^{\text {th }} 75^{\text {th }}\right)$} & \\
\hline SNOT 22 & 48 (33-78.5) & $22.5(12-33.5)$ & 0.047 \\
\hline SNOT 1-12\# & $27(19.5-44)$ & 13.5 (5.2-24.5) & 0.047 \\
\hline Nasal blockage ${ }^{\circ}$ & $4(3-4)$ & $2.5(0.25-3)$ & 0.116 \\
\hline Rhinorrhea ${ }^{\circ}$ & $2.5(2-4)$ & $1.25(0.6-1.9)$ & 0.400 \\
\hline Facial pain ${ }^{\circ}$ & $2(0-4)$ & $0(0-2)$ & 0.133 \\
\hline Ear fullness ${ }^{\circ}$ & $3(0.5-4)$ & $2(0-2.75)$ & 0.519 \\
\hline Hyposmia $^{\circ}$ & $4(2-5)$ & $1(0-4)$ & 0.365 \\
\hline LKS & $6(4.5-8)$ & $4(3.5-8)$ & 0.401 \\
\hline NPS & $3(2-5)$ & $3(2-5)$ & 0.989 \\
\hline \multirow[t]{4}{*}{ Blood eosinophils§ } & 660 (457.5-1162.5) & $490(122.5-532.5)$ & 0.083 \\
\hline & \multicolumn{2}{|c|}{$\begin{array}{c}\text { Anti-IL5 group } \\
\mathrm{n}=20\end{array}$} & \\
\hline & Baseline & 52-week & $P$ \\
\hline & \multicolumn{2}{|c|}{ median (IQR $\left.25^{\text {th }} 75^{\text {th }}\right)$} & \\
\hline SNOT 22 & $64.5(42.7-80.5)$ & $37.5(10.5-55.5)$ & 0.002 \\
\hline SNOT 1-12\# & 36 (25.5-47.2) & 19 (6.7-28) & 0.001 \\
\hline Nasal blockage & $4(3-5)$ & $2(0.75-3)$ & 0.001 \\
\hline Rhinorrhea $^{\circ}$ & $4.5(2.5-5)$ & $2.25(1-3)$ & 0.076 \\
\hline Facial pain ${ }^{\circ}$ & $1(0-5)$ & $0(0-3)$ & 0.127 \\
\hline Ear fullness ${ }^{\circ}$ & $3(1.75-5)$ & $0.5(0-3)$ & 0.026 \\
\hline Hyposmia & $5(0.75-5)$ & $1(0-3.5)$ & 0.068 \\
\hline LKS & $4.5(4-6)$ & $4(2.75-6)$ & 0.253 \\
\hline NPS & $2(2-3)$ & $0(0-2)$ & 0.029 \\
\hline \multirow[t]{4}{*}{ Blood eosinophils§ } & 700 (375-1005) & $80(45-100)$ & 0.001 \\
\hline & \multicolumn{2}{|c|}{$\begin{array}{c}\text { Anti-IL5R group } \\
\mathrm{n}=9\end{array}$} & \\
\hline & Baseline & 52-week & $P$ \\
\hline & \multicolumn{2}{|c|}{ median (IQR $\left.25^{\text {th }} 75^{\text {th }}\right)$} & \\
\hline SNOT 22 & $56(33-70)$ & $24(16-27)$ & 0.063 \\
\hline SNOT 1-12"\# & 35 (26-39) & $13(9-25)$ & 0.024 \\
\hline Nasal blockage ${ }^{\circ}$ & $3(3-4)$ & $1(0-3)$ & 0.222 \\
\hline Rhinorrhea ${ }^{\circ}$ & $2.5(2.2-3)$ & $1(1-2)$ & 0.040 \\
\hline Facial pain & $0(0-4)$ & $0(0-1)$ & 0.666 \\
\hline Ear fullness ${ }^{\circ}$ & $3(0-3)$ & $0(0-2)$ & 0.136 \\
\hline Hyposmia & $5(3-5)$ & $4(0-5)$ & 0.546 \\
\hline LKS & $6(3-6)$ & $4(3-7)$ & $0-796$ \\
\hline NPS & $2(0.5-2)$ & $2(1-3)$ & 0.710 \\
\hline Blood eosinophils§ & $400(350-560)$ & $80(0-287.5)$ & 0.026 \\
\hline
\end{tabular}

SNOT: SinoNasal Outcome Test; LKS: Lund-Kennedy Score; NPS: Nasal Polyp Score \# SNOT 1-12 represents a SNOT 22 sub-category which assesses rhinologic, ear and facial symptoms. SNOT 1-12 value is therefore the sum of the values of the 12 items that composes this subcategory (maximum value 60)

Listed symptoms are individual items of the SNOT 22, each one assessed by a 5-point scale "NPS was calculated only in the CRSWNP subgroup in each treatment arm

$\S$ Blood eosinophils are expressed as number of cells per $\mathrm{mm}^{3}$

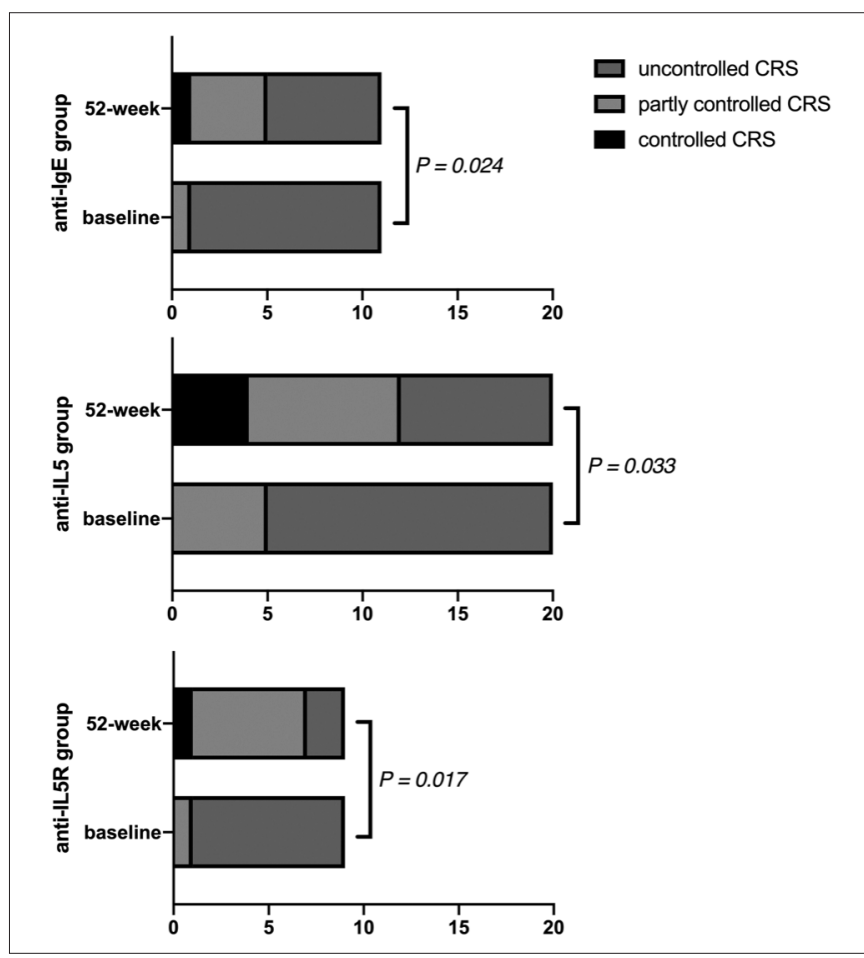

Figure 1. Graphic representation of the degree of nasal control in the three treatment groups and variations between baseline and week 52 .

ence in baseline blood eosinophil count was evident among the three treatment groups $(\mathrm{P}=0.129)$. Differences at Mann-Whitney test between baseline eosinophil count and 52 weeks were significant in the anti-IL5 and anti-IL5R groups $(\mathrm{P}=0.001$ and $\mathrm{P}=0.026$, respectively).

Mean net changes of scores between the three treatment groups along the 52-week observation period are shown in Table V. No significant difference was evident in any of the parameters analysed among the three groups by MannWhitney test (net change SNOT $22 \mathrm{P}=0.951$; net change SNOT $1-12 \mathrm{P}=0.815$; net change LKS $\mathrm{P}=0.565$; net change NPS $\mathrm{P}=0.061$ ).

\section{Discussion}

The treatment of refractory CRS is intensively debated in the literature, especially since biological drugs have shown excellent results in the treatment of asthma. While several RCTs have highlighted the efficacy of mAbs in CRSwNP ${ }^{9-12}$, observational studies evaluating the response to biological treatments in real-life are limited ${ }^{26-30}$.

Although an observational study may have several limitations, the application of clinical trial results to clinical practice is not often straightforward. Issues, such as restrictive 
Table V. Differences in net change of selected scores between treatment groups.

\begin{tabular}{|c|c|c|c|}
\hline & $\begin{array}{c}\text { Anti-lgE } \\
\text { group } \\
n=11 \\
\text { mean } \pm \text { SD }\end{array}$ & $\begin{array}{c}\text { Anti-IL5 } \\
\text { group } \\
n=20 \\
\text { mean } \pm \text { SD }\end{array}$ & $\begin{array}{c}\text { Anti-IL5R } \\
\text { group } \\
n=9 \\
\text { mean } \pm \text { SD }\end{array}$ \\
\hline Net change SNOT 22 & $24.6 \pm 27.1$ & $26.9 \pm 20.6$ & $25.3 \pm 26.9$ \\
\hline Net change SNOT 1-12 & $14 \pm 14.3$ & $16.9 \pm 10.6$ & $14.7 \pm 16.3$ \\
\hline Net change LKS & $1.0 \pm 1.5$ & $0.2 \pm 2.8$ & $0.4 \pm 2.5$ \\
\hline Net change NPS* & $0.1 \pm 0.8$ & $0.8 \pm 1.4$ & $-0.3 \pm 1.2$ \\
\hline
\end{tabular}

SNOT: SinoNasal Outcome Test; LKS: Lund-Kennedy Score; NPS: Nasal Polyp Score

Net change NPS was calculated only in the CRSWNP subgroup in each treatment arm.

enrollment criteria, experimental design limitations, conflicts of interest (both financial and non-financial), publication bias and biological variability, can all underlie the disparity between the outcomes achieved from clinical trials compared to those observed in real-life ${ }^{31}$.

For instance, exclusion criteria from clinical trials encompass the absence of nasal polyps or NPS lower than $5^{12}$, continuative use of high-dose OCS, treatment with another biologic in the previous 12 months, asthma exacerbations requiring hospitalisation within the period of screening ${ }^{12}$, OCS and surgical intervention from 1 month before treatment until the end of the study, and use of nasal steroid therapies for two months after first dose ${ }^{10}$.

Contrarily, the presented study group included both patients with CRSwNP (82.5\%) and CRSsNP (17.5\%); patients with nasal polyps had a baseline mean NPS of $2.6 \pm 1.9$; 6 patients (15\%) underwent a sinonasal surgical procedure during $\mathrm{mAb}$ therapy, 18 patients $(45 \%)$ were in chronic OCS treatment for control of airway exacerbations and 26 patients $(65 \%)$ were in chronic nasal steroid therapy. In addition, a change in mAb therapy was observed in 7 patients in order to achieve better control of the lower airways: 5 patients $(12.5 \%)$ switched from omalizumab to mepolizumab and 2 patients (5\%) from mepolizumab to benralizumab at the time of enrollment in our study.

It follows that, considering the aforementioned exclusion criteria, most of our patients would not have been suitable for a RCT. All of the above, together with the associated non-respiratory comorbidities, explains the extreme variability of patients encountered in the daily practice.

Another element that further enriches our knowledge on the effectiveness of mAbs is the long-term follow-up. Our 52-week observation goes beyond previous literature experiences which evaluated patients at shorter times (16 weeks for omalizumab ${ }^{11}$ and 25 weeks for mepolizumab ${ }^{12}$ ), providing a longer perspective on the effects of biological therapies in CRS.

Most of the RCTs published in the literature select endo- scopic changes of nasal polyps as the primary outcome of treatment.

Gevaert et al. ${ }^{11}$ reported a significant reduction in polyp size compared to baseline in the omalizumab group $(\mathrm{P}=0.001)$, which also presented a significantly lower NPS than the placebo arm throughout the entire treatment period $(\mathrm{P}=0.02)$. Another study by Gevaert et al. ${ }^{10}$ reported a significant reduction of NPS in the mepolizumab group compared to placebo $(\mathrm{P}=0.028)$. Bachert et al. ${ }^{12}$ showed a significant higher probability of having a reduction of NPS in the mepolizumab group vs. placebo $(\mathrm{P}=0.031)$. Differently, Pinto et al. ${ }^{9}$ opted for changes in sinus opacification determined by CT scan as the primary outcome, reporting a significant reduction in inflammation for the omalizumab group from baseline $(\mathrm{P}=0.043)$, without significant differences on net change across treatment groups $(P=0.391)$. NPS from baseline and net change across treatments did not show significant variations $(\mathrm{P}=0.58)$.

Our evaluation of endoscopic parameters showed no significant differences in LKS $(\mathrm{P}=0.124)$ and NPS $(\mathrm{P}=0.130)$ between baseline and 52 weeks in the entire cohort; moreover, even the net change appeared quite small. Although, at a first glance, only anti-IL5 therapy was able to reduce NPS, this result should be considered with caution as it was strongly influenced by combination with surgical approaches.

It is proper to underline that mean baseline NPS in our cohort was clearly lower than in the aforementioned RCTs. This leads to the consideration that mAbs might be effective in polyp volume reduction, but not in their full reabsorption, so that beyond a certain shrinking threshold there is no further clinical improvement. Moreover, nasal polyp formation is known to depend on the complex interplay among different mechanisms orchestrated by a much wider panel of molecules than IgE or IL5. Likewise, the fact that LKS did not improve might imply that the impact of these specific drugs on nasal tissue remodeling in a broader sense (related to mucin production, extracellular matrix modifications, epithelial barrier functions) is somewhat limited ${ }^{32}$. Secondary endpoints included, among others, nasal symptoms. Pinto et al. ${ }^{9}$ reported significant improvement in SNOT 20 with omalizumab $(\mathrm{P}<0.05)$ from baseline, but there was no difference in the net change between omalizumab and placebo $(\mathrm{P}<0.78)$; Gevaert et al. ${ }^{11}$ reported a significant decrease in symptom scores for nasal congestion $(\mathrm{P}=0.002)$, anterior rhinorrhoea $(\mathrm{P}=0.003)$, hyposmia $(\mathrm{P}=0.004)$, wheeze $(\mathrm{P}=0.02)$ and dyspnoea $(\mathrm{P}=0.02)$ and a significant improvement of Short-Form Health Questionnaire 36 (SF-36) in the omalizumab group $(\mathrm{P}=0.02)$; Gevaert et al. ${ }^{10}$ reported a non-statistically significant improvement of smell, postnasal drip and conges- 
tion in patients treated with mepolizumab, while Bachert et al. ${ }^{12}$ reported greater improvement with mepolizumab compared to placebo for SNOT $22(\mathrm{P}=0.005)$ and individual symptoms such as rhinorrhoea $(\mathrm{P}<0.001)$, mucus in throat $(\mathrm{P}<0.001)$, nasal blockage $(\mathrm{P}=0.002)$ and loss of smell $(\mathrm{P}<0.001)$.

In our study, an improvement of symptoms was evident with a significant reduction of SNOT $22(\mathrm{P}<0.001)$, SNOT $1-12(\mathrm{P}<0.001)$ and individual symptoms such as nasal blockage $(\mathrm{P}<0.001)$, rhinorrhoea $(\mathrm{P}<0.021)$, hyposmia $(\mathrm{P}=0.023)$, facial pain $(\mathrm{P}=0.027)$ and ear fullness $(P=0.006)$. However, subgroup analysis showed similar improvements only for some of these symptoms and not shared by all treatment arms. It is reasonable to think that this discrepancy is mainly due to the limited number of individuals in each subgroup.

It clearly emerges from these comparisons that, in real-life, the classic primary endpoint of RCTs was not achieved, even though overall symptom improvement was statistically significant. One possible explanation is that patients treated with mAbs for asthma, in which a significant reduction in pulmonary exacerbations and an overall improvement of lower airways obstruction occurs, may also perceive an improvement in nasal symptoms that is not necessarily related to objective nasal findings ${ }^{33,34}$.

A further consideration is unavoidable. It is reasonable to ask whether the only improvement in patient-reported symptoms is sufficient to define a good outcome or whether the lack of a significant improvement in endoscopic findings may be related to a faulty choice of mAb. In other words, are we satisfied only with improvement in symptoms or should we consider changing therapy when no endoscopic improvement is observed? According to current guidelines, a "moderate response" to a biological treatment (fulfillment of 3-4 criteria) would theoretically be achieved regardless of variations in nasal polyp size and only an "excellent response" would be defined on the fulfillment of all 5 evaluation criteria ${ }^{17}$.

Coupling the objective and subjective aspects of the disease, together with other items as proposed by the EPOS multimodal scale for CRS control (i.e. sleep disturbance or fatigue, systemic medications needed to control disease) ${ }^{17,18}$, we were able to show a significant difference on control of nasal disease between baseline and 52 weeks in all treatment groups. Interestingly, although all patients in the cohort were (for obvious reasons) affected by severe asthma, we found that $17.5 \%$ of patients presented with a partly controlled CRS at baseline. This suggests the possible coexistence of mild or moderate nasal disease in patients with severe lower airways comorbidity and, at the same time, the potential association of severe forms of CRS with mild or moderate asthma (unpublished data). Moreover, patients presenting with this latter combination are penalised because they are currently beyond the prescriptive limits of mAbs therapy in Italy.

Some reflections arise about the degree of nasal disease control as proposed by EPOS: the presence of particular symptoms, such as refractory smell loss and rhinorrhoea, is very common in all CRS stages, despite good overall control of disease; sleep disturbance or fatigue may be related to other general conditions that are not strictly connected to CRS; endoscopic findings of healthy or almost healthy mucosa is limited in clinical practice to very mild forms of CRS. This would mean that a revised and more accurate definition of disease control might down-stage patients defined as uncontrolled and not responsive to treatments.

No serious AEs were reported in the entire cohort, but minor AEs occurred in 25 patients $(62.5 \%)$. The most common were nasopharyngitis $(42.5 \%)$, oropharyngeal pain $(37.5 \%)$ and back pain (27.5\%). These findings appear concordant with previous reports ${ }^{10}$.

The limits of our study first encompass the small sample size and its diversity in terms of patient characteristics and treatments. Larger and more uniform series are needed to verify these preliminary results.

Second, all patients were on mAb treatment and therefore we lack a control group.

Subgroup analysis between CRSwNP and CRSsNP, although conceptually consistent with the indications for the use of biological drugs in CRS, was not conducted because the size of the CRSsNP sample was too small for significant statistical considerations. In the literature, this dichotomous classification is increasingly cornered in favour of an endotype-based definition from which it emerges that these two phenotypes may share the same inflammatory mechanisms ${ }^{35}$ and, thus, be potentially manageable in a similar manner.

We have shortcomings regarding biological parameters: peripheral eosinophil count was missing for some patients, peripheral IgE were dosed only once before starting treatment in the anti-IgE group, other biomarkers of type 2 inflammation (i.e. tissue eosinophil count, serum periostin, exhaled and nasal nitric oxide) were unavailable.

Moreover, we were not able to assess radiologic changes after treatment. Indeed, CT scan are repeated in daily practice only in refractory cases in expectation of a surgical procedure or a clinical need.

Although all patients self-reported an overall improvement of asthma-related quality of life, this feedback was not verified through validated questionnaires.

Lastly, we did not extensively evaluate the impact of surgery on outcomes; although mAb treatment groups did not 
differ in the prevalence of previous endoscopic sinus surgery $(\mathrm{P}=0.431)$, the variability in the type of surgical procedure did not allow comparisons between patients. For the same reason, thorough endoscopic assessment of outcomes of sinus surgery, which include the evaluation of patency of ostia, presence of scarring, characteristics of nasal secretions (other than LKS items) and microbiological data, was not reported.

\section{Conclusions}

The upcoming introduction of biologics in clinical practice may represent a promising treatment for refractory CRS. However, many issues are still open. Among these, RCTs published to date focus only on the CRSwNP phenotype, while CRSsNP are also known to present as a consequence of similar inflammatory pathways.

The present observational study showed an improvement of nasal symptoms in long-term follow-up that was not associated with significant variations in endoscopic findings. These preliminary results, of course, need to be verified in more extensive real-life studies. Moreover, we still lack data about the cost-effectiveness of these therapies, their relationship with surgery (interdependent or exclusive) and an accurate set of selection criteria and predictive biomarkers. Lastly, we do not exclude that in the near future other treatment options, upstream in the inflammatory cascade, will be developed that target the entire spectrum of mechanisms underlying multifactorial diseases, like CRS and asthma.

\section{Acknowledgements}

Andrea Preti is a PhD student of the "Sperimental and translational medicine" course at University of Insubria.

\section{References}

1 Schleimer RP. Immunopathogenesis of chronic rhinosinusitis and nasal polyposis. Annu Rev Pathol 2017;12:331-57. https://doi. org/10.1146/annurev-pathol-052016-100401

2 Cao PP, Wang ZC, Schleimer RP, et al. Pathophysiologic mechanisms of chronic rhinosinusitis and their roles in emerging disease endotypes. Ann Allergy Asthma Immunol 2019;122:33-40. https://doi. org/10.1016/j.anai.2018.10.01

3 Krouse JH, Krouse HJ. Asthma, rhinitis, and the unified airway. ORL Head Neck Nurs 2013;31:6-10.

4 van der Veen J, Seys SF, Timmermans M, et al. Real-life study showing uncontrolled rhinosinusitis after sinus surgery in a tertiary referral centre. Allergy 2017;72:282-90.https://doi.org/10.1111/all.12983

5 Calus L, Van Bruaene N, Bosteels C, et al. Twelve-year follow-up study after endoscopic sinus surgery in patients with chronic rhinosinusitis with nasal polyposis. Clin Transl Allergy 2019;9:30. https:// doi.org/10.1186/s13601-019-0269-4

6 Edris A, De Feyter S, Maes T, et al. Monoclonal antibodies in type 2 asthma: a systematic review and network meta-analysis. Respir Res 2019;20:179. https://doi.org/10.1186/s12931-019-1138-3

7 Ren L, Zhang N, Zhang L, et al. Biologics for the treatment of chronic rhinosinusitis with nasal polyps - state of the art. World Allergy Organ J 2019;12:100050. https://doi.org/10.1016/j.waojou.2019.100050

8 Kartush AG, Schumacher JK, Shah R, et al. Biologic agents for the treatment of chronic rhinosinusitis with nasal polyps. Am J Rhinol Allergy 2019;33:203-11. https://doi.org/10.1177/1945892418814768

9 Pinto JM, Mehta N, Di Tineo M, et al. A randomized, double-blind, placebo-controlled trial of anti-IgE for chronic rhinosinusitis. Rhinology 2010;48:318-24. https://doi.org/10.4193/Rhin09.144

10 Gevaert P, Van Bruaene N, Cattaert T, et al. Mepolizumab, a humanized anti-IL-5 mAb, as a treatment option for severe nasal polyposis. J Allergy Clin Immunol 2011;128:989-95.e1-8. https://doi. org/10.1016/j.jaci.2011.07.056

11 Gevaert P, Calus L, Van Zele T, et al. Omalizumab is effective in allergic and nonallergic patients with nasal polyps and asthma. J Allergy Clin Immunol 2013;131:110-6.e1. https://doi.org/10.1016/j. jaci.2012.07.047

12 Bachert C, Sousa AR, Lund VJ, et al. Reduced need for surgery in severe nasal polyposis with mepolizumab: Randomized trial. J Allergy Clin Immunol 2017;140:1024-31.e14. https://doi.org/10.1016/j. jaci.2017.05.044

13 Global Initiative for Asthma. Global strategy for asthma management and prevention. 2017. Available from: www.ginasthma.org

14 Global Initiative for Asthma. Global strategy for asthma management and prevention. 2018. Available from: www.ginasthma.org

15 Global Initiative for Asthma. Global strategy for asthma management and prevention. 2019. Available from: www.ginasthma.org

16 Global Initiative for Asthma. Global strategy for asthma management and prevention. 2020. Available from: www.ginasthma.org

17 Fokkens WJ, Lund VJ, Hopkins C, et al. European position paper on rhinosinusitis and nasal polyps 2020. Rhinology 2020;58(Suppl S29):1-464. https://doi.org/10.4193/Rhin20.600

18 Fokkens WJ, Lund VJ, Mullol J, et al. European position paper on rhinosinusitis and nasal polyps 2012. Rhinol Suppl 2012;23:1-298.

19 Castelnuovo P, Bandi F, Preti, et al. Implementing strategies for data collection in chronic rhinosinusitis. Acta Otorhinolaryngol Ital 2018;38:222-4. https://doi.org/10.14639/0392-100X-1993

20 Akdis CA, Hellings, PW, Agache I; European Academy of Allergy and Clinical Immunology, Editors. Global atlas of allergic rhinitis and chronic rhinosinusitis. Zurich, Switzerland: European Academy of Allergy and Clinical Immunology; 2015.

21 Mozzanica F, Preti A, Gera R, et al. Cross-cultural adaptation and validation of the SNOT-22 into Italian. Eur Arch Otorhinolaryngol 2017;274:887-95. https://doi.org/10.1007/s00405-016-4313-x

22 Gallo S, Russo F, Mozzanica F, et al. Prognostic value of the Sinonasal Outcome Test 22 (SNOT-22) in chronic rhinosinusitis. Acta Otorhinolaryngol Ital 2020;40:113-21. https://doi.org/10.14639/0392-100XN0364

23 Gelardi M, Piccininni K, Quaranta N, et al. Olfactory dysfunction in patients with chronic rhinosinusitis with nasal polyps is associated with clinical-cytological grading severity. Acta Otorhinolaryngol Ital 2019;39:329-35. https://doi.org/10.14639/0392-100X-2426

24 Lund VJ, Kennedy DW. Staging for rhinosinusitis. Otolaryngol Head Neck Surg 1997;117(3 Pt 2):S35-40. https://doi.org/10.1016/s01945998(97)70005-6

25 Meltzer EO, Hamilos DL, Hadley JA. Rhinosinusitis: developing guidance for clinical trials. J Allergy Clin Immunol 2006;118(5 Suppl):S17-61. https://doi.org/10.1016/j.jaci.2006.09.005

26 Bidder T, Sahota J, Rennie C, et al. Omalizumab treats chronic rhi- 
nosinusitis with nasal polyps and asthma together-a real life study. Rhinology 2018;56:42-5. https://doi.org/10.4193/Rhin17.139

27 Numata T, Nakayama K, Utsumi H, et al. Efficacy of mepolizumab for patients with severe asthma and eosinophilic chronic rhinosinusitis. BMC Pulm Med 2019;19:176. https://doi.org/10.1186/s12890019-0952-1

28 Tsurumaki H, Matsuyama T, Ezawa K, et al. Rapid effect of benralizumab for hypereosinophilia in a case of severe asthma with eosinophilic chronic rhinosinusitis. Medicina (Kaunas) 2019;55:336. https:// doi.org/10.3390/medicina55070336

29 Rowan NR, Naclerio RM. Persistence of sinonasal disease despite mepolizumab. J Allergy Clin Immunol Pract 2020;8:1550-5. https:// doi.org/10.1016/j.jaip.2020.01.049

30 Jandus P, Harr T, Soyka MB, et al. Efficacité de l'omalizumab dans la polypose nasale: à propos de deux cas [The efficacy of omalizumab in the treatment of chronic rhinosinusitis with nasal polyps: a discussion of 2 refractory cases]. Rev Med Suisse 2019;15:1748-51.
31 Zarbin M. Real life outcomes vs. clinical trial results. J Ophthalmic Vis Res 2019;14:88-92. https://doi.org/10.4103/jovr.jovr_279_18

32 Shay AD, Tajudeen BA. Histopathologic analysis in the diagnosis and management of chronic rhinosinusitis. Curr Opin Otolaryngol Head Neck Surg 2019;27:20-4. https://doi.org/10.1097/ MOO.0000000000000510

33 Huang CC, Chang PH, Wu PW, et al. Impact of nasal symptoms on the evaluation of asthma control. Medicine (Baltimore) 2017;96:e6147. https://doi.org/10.1097/MD.0000000000006147

34 Chan R, RuiWen Kuo C, Lipworth B. Disconnect between effects of mepolizumab on severe eosinophilic asthma and chronic rhinosinusitis with nasal polyps. J Allergy Clin Immunol Pract 2020;8:1714-6. https://doi.org/10.1016/j.jaip.2020.01.009

35 Delemarre T, Holtappels G, De Ruyck N, et al. Type 2 inflammation in chronic rhinosinusitis without nasal polyps: another relevant endotype. J Allergy Clin Immunol 2020;146:337-43.e6. https://doi. org/10.1016/j.jaci.2020.04.040 